\title{
Nonlinear Dirac equations on Riemann surfaces
}

\author{
Qun Chen · Jürgen Jost • Guofang Wang
}

Received: 19 April 2007 / Accepted: 13 July 2007 / Published online: 11 August 2007

(C) Springer Science+Business Media B.V. 2007

\begin{abstract}
We have developed analytical methods for nonlinear Dirac equations. Examples of such equations include Dirac-harmonic maps with curvature term and the equations describing the generalized Weierstrass representation of surfaces in three-manifolds. We have provided the key analytical steps, i.e., small energy regularity and removable singularity theorems and energy identities for solutions.
\end{abstract}

Keywords Dirac equation $\cdot$ Regularity $\cdot$ Energy identity

Mathematics Subject Classifications (2000) $\quad 58 \mathrm{~J} 05 \cdot 53 \mathrm{C} 27$

\section{Introduction}

Dirac type equations on Riemann surfaces are ubiquitous in geometry, as they constitute the most basic first order system of elliptic equations. The first examples are of course the Cauchy-Riemann equations. These are linear, but other examples are typically of the nonlinear type

$$
\not \partial \psi=H_{j k l}\left\langle\psi^{j}, \psi^{k}\right\rangle \psi^{l}
$$

Communicated by H.-B. Rademacher (Leipzig).

Q. Chen

School of Mathematics and Statistics, Wuhan University, Wuhan 430072, China

e-mail: qunchen@whu.edu.cn

J. Jost $(\bowtie)$

Max Planck Institute for Mathematics in the Sciences, Inselstr. 22, Leipzig 04103, Germany

e-mail: jjost@mis.mpg.de

G. Wang

Faculty of Mathematics, University Magdeburg, Magdebrug 39016, Germany

e-mail: gwang@math.uni-magdeburg.de 
with a notation to be explained shortly. The linear operator on the left-hand side is, of course, the Dirac operator whereas the nonlinearity on the right-hand side is cubic. As we shall see, this type of nonlinearity on one hand arises naturally in geometry, because (1.1) is conformally invariant and on the other hand, from the analytical side, it presents a borderline case, where standard linear methods fail to apply (again, because it is conformally invariant), but an analytical treatment nevertheless is still possible by utilizing the structure of the equation in a more sophisticated manner. That is, analytical methods need to be supplemented by geometric insights. This frame makes (1.1) particularly attractive. In the present article, we develop a systematic and general treatment of the key steps of the nonlinear analysis.

Let us now describe the underlying geometric structure in more detail. Let $(M, g)$ be a Riemann surface with a fixed spin structure, and denote the spin bundle by $\Sigma$. On $\Sigma$, there is a Hermitian metric $\langle\cdot, \cdot\rangle$ compatible with the spin connection $\nabla$ on $\Sigma$. For any orthonormal basis $\left\{e_{\alpha}, \alpha=1,2\right\}$ on $M$, the (Atiyah-Singer) Dirac operator is defined by $\not \partial:=e_{\alpha} \cdot \nabla_{e_{\alpha}}$, where . stands for the Clifford multiplication. In this article, we use the summation convention.

We consider (1.1), that is,

$$
\not \partial \psi=H_{j k l}\left\langle\psi^{j}, \psi^{k}\right\rangle \psi^{l}
$$

where $\psi=\left(\psi^{1}, \psi^{2}, \ldots, \psi^{n}\right) \in \Gamma\left(\Sigma^{n}\right), \Sigma^{n}:=\overbrace{\Sigma \times \cdots \times \Sigma}^{n}, n \in \mathbb{Z}_{+}$and $H_{j k l}=$ $\left(H_{j k l}^{1}, H_{j k l}^{2}, \ldots, H_{j k l}^{n}\right) \in C^{1}\left(M, \mathbb{R}^{n}\right)$. Denote $h_{0}:=\max \left\{\left|H_{j k l}^{i}\right|(x) ; x \in M, i, j, k, l=\right.$ $1,2, \ldots, n\}, h_{1}:=\max \left\{\left|\nabla H_{j k l}^{i}\right|(x) ; x \in M, i, j, k, l=1,2, \ldots, n\right\}$ and $|\psi|:=$ $\left(\sum_{i=1}^{n}\left\langle\psi^{i}, \psi^{i}\right\rangle\right)^{1 / 2}$. We note that (1.2) is conformally invariant.

Let us now discuss examples where (1.2) arises. In fact, we have been led to it through our study of Dirac-harmonic maps with curvature term (c.f. [6]) which in turn were derived from the nonlinear supersymmetric $\sigma$-model of quantum field theory, where Dirac type equations describe fermionic particles. Let $\phi$ be a smooth map from $M$ to a Riemannian manifold $(N, h)$ of dimension $n \geq 2$ and $\phi^{-1} T N$ the pull-back bundle of $T N$ by $\phi$. On the twisted bundle $\Sigma \otimes \phi^{-1} T N$, there is a metric (also denoted by $\langle\cdot, \cdot\rangle$ ) and a natural connection $\widetilde{\nabla}$ induced from those on $\Sigma$ and $\phi^{-1} T N$. In local coordinates $\left\{x_{\alpha}\right\}$ and $\left\{y^{i}\right\}$ on $M$ and $N$ respectively, a section $\psi$ of $\Sigma \otimes \phi^{-1} T N$ takes the form

$$
\psi(x)=\psi^{j}(x) \otimes \partial_{y^{j}}(\phi(x))
$$

and $\widetilde{\nabla}$ can be written as

$$
\widetilde{\nabla} \psi(x)=\nabla \psi^{i}(x) \otimes \partial_{y^{i}}(\phi(x))+\Gamma_{j k}^{i}(\phi(x)) \nabla \phi^{j}(x) \psi^{k}(x) \otimes \partial_{y^{i}}(\phi(x)),
$$

where $\psi^{i} \in \Gamma(\Sigma),\left\{\partial_{y^{j}}\right\}$ is the natural local basis on $N$ and $\left\{\Gamma_{j k}^{i}\right\}$ stands for the Christoffel symbols of $N$. The Dirac operator along the map $\phi$ is defined as

$$
\begin{aligned}
\not D \psi & :=e_{\alpha} \cdot \widetilde{\nabla}_{e_{\alpha}} \psi \\
& =\not \partial \psi^{i}(x) \otimes \partial_{y^{i}}(\phi(x))+\Gamma_{j k}^{i}(\phi(x)) \nabla_{e_{\alpha}} \phi^{j}(x) e_{\alpha} \cdot \psi^{k}(x) \otimes \partial_{y^{i}}(\phi(x)) .
\end{aligned}
$$

In [6], we considered the following functional:

$$
L_{c}(\phi, \psi):=\frac{1}{2} \int_{M}\left[|\mathrm{~d} \phi|^{2}+\langle\psi, \not D \psi\rangle-\frac{1}{6} R_{i k j l}\left\langle\psi^{i}, \psi^{j}\right\rangle\left\langle\psi^{k}, \psi^{l}\right\rangle\right] .
$$

We call critical points $(\phi, \psi)$ of $L_{c}$ Dirac-harmonic maps with curvature term. This functional is dictated by the supersymmetry requirements of the $\sigma$-model in superstring theory. The difference is that, here the components of $\psi$ are ordinary spinor fields on $M$, while 
in physics they take values in a Grassmann algebra. The Euler-Lagrange equations of the functional $L_{c}$ are (see [6] for details):

$$
\begin{gathered}
\tau^{i}(\phi)-\frac{1}{2} R^{i}{ }_{l m j}\left\langle\psi^{m}, \nabla \phi^{l} \cdot \psi^{j}\right\rangle+\frac{1}{12} h^{i p} R_{m k j l ; p}\left\langle\psi^{m}, \psi^{j}\right\rangle\left\langle\psi^{k}, \psi^{l}\right\rangle=0, \\
\not \psi^{i}=-\frac{1}{3} R^{i}{ }_{j k l}\left\langle\psi^{j}, \psi^{k}\right\rangle \psi^{l}, \quad i=1,2, \ldots, n,
\end{gathered}
$$

where $\tau(\phi)$ is the tension field of $\phi, R^{i}{ }_{j k l}$ stands for a component of the curvature tensor of $N$ and $R_{m k j l ; p}$ denotes the covariant derivative of $h_{m i} R^{i}{ }_{k j l}$ with respect to $\partial_{y} p$.

In particular, if $\phi$ is a constant map, then (1.5) becomes

$$
\not \partial \psi^{i}=-\frac{1}{3} R^{i}{ }_{j k l}\left\langle\psi^{j}, \psi^{k}\right\rangle \psi^{l}, \quad i=1,2, \ldots, n,
$$

which is a Dirac equation of type (1.2).

Another more classical example of an equation of type (1.2) comes from the geometry of surfaces in three-manifolds via the generalized Weierstrass representation, as we shall now explain. When $n=1$, it takes the form

$$
\not \partial \psi=H|\psi|^{2} \psi
$$

for $H \in C^{1}(M), \psi \in \Gamma(\Sigma)$. A similar equation was considered by Ammann and Humbert in [2] when they studied the first conformal Dirac eigenvalue. See also [1] for a Yamabe type problem.

Recall the classical Weierstrass formula that represents minimal surfaces $X$ immersed in $\mathbb{R}^{3}$ in terms of a holomorphic 1 -form and a meromorphic function. The generalized Weierstrass representation was found to express a general surface immersed in $\mathbb{R}^{3}$ (as well as in $\mathbb{R}^{4}$ and some three-dimensional Lie groups) by Dirac equations (see e.g. $[8,11,16]$ ). For the ambient space $\mathbb{R}^{3}$, a surface $X: M \rightarrow \mathbb{R}^{3}$ is represented by

$$
X=\operatorname{Re} \int\left(i\left(\psi_{1}^{2}+\bar{\psi}_{2}^{2}\right), \bar{\psi}_{2}^{2}-\psi_{1}^{2}, 2 \psi_{2} \bar{\psi}_{2}\right)
$$

where $\psi:=\left(\begin{array}{l}\psi_{1} \\ \psi_{2}\end{array}\right): \mathbb{R}^{2} \rightarrow \mathbb{C}^{2}$ satisfies the following equation:

$$
\left[2\left(\begin{array}{cc}
0 & \bar{\partial} \\
-\partial & 0
\end{array}\right)+\left(\begin{array}{cc}
U & 0 \\
0 & U
\end{array}\right)\right]\left(\begin{array}{l}
\psi_{1} \\
\psi_{2}
\end{array}\right)=0,
$$

with $U=H|\psi|^{2}$. On the Euclidean plane $\mathbb{R}^{2}$ the spin structure is unique, and the spinor bundle $\Sigma$ is trivial. By choosing a representation of $\left\{e_{\alpha}\right\}$ as

$$
\sigma_{1}=\left(\begin{array}{cc}
0 & 1 \\
-1 & 0
\end{array}\right), \quad \sigma_{2}=\left(\begin{array}{ll}
0 & i \\
i & 0
\end{array}\right), i:=\sqrt{-1}
$$

the Dirac operator can be expressed as

$$
\begin{aligned}
\not \partial \psi & =\left(\begin{array}{cc}
0 & 1 \\
-1 & 0
\end{array}\right)\left(\begin{array}{l}
\partial_{x} \psi_{1} \\
\partial_{x} \psi_{2}
\end{array}\right)+\left(\begin{array}{ll}
0 & i \\
i & 0
\end{array}\right)\left(\begin{array}{l}
\partial_{y} \psi_{1} \\
\partial_{y} \psi_{2}
\end{array}\right) \\
& =2\left(\begin{array}{cc}
0 & \bar{\partial} \\
-\partial & 0
\end{array}\right)\left(\begin{array}{l}
\psi_{1} \\
\psi_{2}
\end{array}\right),
\end{aligned}
$$


where $\psi:=\left(\begin{array}{l}\psi_{1} \\ \psi_{2}\end{array}\right): \mathbb{R}^{2} \rightarrow \mathbb{C}^{2}$ is a spinor field, $\partial:=\frac{1}{2}\left(\partial_{x}-i \partial_{y}\right), \bar{\partial}:=\frac{1}{2}\left(\partial_{x}+i \partial_{y}\right)$. Therefore, Eq. 1.8 can be written as (1.7) which is a special case of (1.2) with $n=1$. Similar types of equations will be discussed in Sect. 5 .

We now turn to the analytical aspects and introduce the following energy functional:

$$
E(\psi):=\int_{M}|\psi|^{4} .
$$

For the analysis of the equation (1.2), we use $\|\psi\|_{L^{4}}$, instead of $\|\nabla \psi\|_{L^{\frac{4}{3}}}$, as the energy functional of $\psi$, since the former is strong enough to get various estimates in most cases, as one can see in [4] and [5].

For a solution $\psi$ of (1.2), if $\psi \in L^{r}$ for some $r>4$, then the standard argument of elliptic regularity theory implies the smoothness of $\psi$. Furthermore, under the condition of uniformly bounded $L^{r}(r>4)$ norms, one has compactness for these solutions. However, if we only assume the boundedness of the $L^{4}$ norm of $\psi$, then the compactness is no longer true. One then naturally considers the blow up phenomenon for the solutions. In particular, it is interesting to know whether the energy identity and removable singularity results hold. $\psi \in L^{4}$ is the borderline case for the geometric analysis of the solutions of (1.2), since the standard bootstrap method in elliptic estimates fails in the first step. It turns out that this can be overcome by establishing some $L^{p}$ elliptic boundary estimates and combining various estimates in delicate ways.

In this article, our aim is to study properties of the solution space of this and similar types of equations. We will prove small energy regularity theorems in which basic a priori estimates for smooth solutions of (1.2) and related types of equations are given. Then we prove a removable singularity theorem, which provides a key tool for the blow up analysis of the solutions. Based on these, we consider the blow up process of solutions under a uniform bound for the $L^{4}$ norm, and we will establish an energy identity for these solutions. For harmonic maps, holomorphic curves and also maps with uniformly $L^{2}$-bounded tension fields, these results are derived in [7,10,13-15,17].

We would like to remark that these considerations are closely related to the regularity of weak solutions, corresponding to the well-known case of harmonic maps in dimension two. Based on the discussion in this article, we believe that, using an $L^{p}$ theory for boundary value problems of the Dirac equations (see [3] for the $L^{2}$ theory), regularity results for weak solutions are available.

\section{Small energy regularity theorem}

In this section, we will prove a small energy regularity theorem. Since the problem is local and Eq. 1.2 is conformally invariant, without loss of generality, we may assume $M$ to be the unit disk

$$
B=\left\{(x, y) \in \mathbb{R}^{2} \mid x^{2}+y^{2}<1\right\}
$$

equipped with the Euclidean metric.

Theorem 2.1 (Small energy regularity theorem) There exists a small constant $\varepsilon>0$ such that for any smooth solution $\psi$ of (1.2) satisfying

$$
E(\psi ; B):=\int_{B}|\psi|^{4}<\varepsilon,
$$


we have

$$
\|\psi\|_{B^{\prime}, k, p} \leq C\|\psi\|_{B, 0,4},
$$

$\forall B^{\prime} \subset \subset B, 1<p$ and $k \in \mathbb{Z}_{+}$, where $C=C\left(B^{\prime}, k, p\right)>0$ is a constant, and $\|\cdot\|_{B, k, p}$ denotes the norm in $W^{k, p}\left(B, \Sigma^{n}\right)$.

For proving this result, we need the following $L^{p}$ boundary estimates for Dirac operators. This is essentially Lemma 4.8 in [4], but we will give another proof here.

Lemma 2.2 Suppose $\psi$ is a solution of

$$
\not \partial=f
$$

on $B$, with $\left.\psi\right|_{\partial B}=\varphi$, and $f \in L^{p}\left(B, \Sigma^{n}\right), \varphi \in W^{1, p}\left(\partial B, \Sigma^{n}\right)$ for some $p>1$, then

$$
\|\psi\|_{B, 1, p} \leq C\left(\|f\|_{B, 0, p}+\|\varphi\|_{\partial B, 1, p}\right),
$$

where $C=C(p)>0$ is a constant.

Proof of Lemma 2.2 First, let

$$
w(x):=\int_{B} \Gamma(x-y) f(y) \mathrm{d} y
$$

be the Dirac-Newton potential of $f$, where

$$
\Gamma(x):=-\frac{1}{2 \pi} \frac{x}{|x|^{2}} .
$$

is the Green function of $\not \partial$. Using the relation between $\Gamma$ and the Green function $\widetilde{\Gamma}$ of the Laplace operator: $\Gamma=\not \partial \widetilde{\Gamma}$, one sees that $w=\not \partial \widetilde{w}$, where

$$
\tilde{w}(x):=\int_{B} \widetilde{\Gamma}(x-y) f(y) \mathrm{d} y .
$$

From the Calderon-Zygmund inequality (see e.g. [9] Theorem 9.9):

$$
\left\|\nabla^{2} \tilde{w}\right\|_{B, 0, p} \leq C\|f\|_{B, 0, p} .
$$

Noting that

$$
\nabla_{\alpha} w=\nabla_{\alpha} \not \tilde{w}=\sigma_{\beta} \nabla_{\alpha} \nabla_{\beta} \tilde{w}
$$

it follows that

$$
\|\nabla w\|_{B, 0, p} \leq C\|f\|_{B, 0, p} .
$$

Second, for any $\xi \in W_{0}^{1, p}\left(B, \Sigma^{n}\right)$ and $F \in L^{p}\left(B, \Sigma^{n}\right)$ satisfying

$$
\not \partial \xi=F,
$$

we have

$$
\|\nabla \xi\|_{B, 0, p} \leq\|F\|_{B, 0, p}
$$


In fact, there exists a sequence of $\xi_{k} \in C_{0}^{1}\left(B, \Sigma^{n}\right)$ such that $\xi_{k} \rightarrow \xi$ in $W^{1, p}$ which implies $\not \partial \xi_{k} \rightarrow \not \partial \xi$ in $L^{p}$. Denote $F_{k}:=\not \partial \xi_{k}$, since $\xi_{k}$ has compact support, we know (see, e.g., [12]) that $\xi_{k}$ is the Dirac-Newton potential of $F_{k}$, and by (2.5) we have

$$
\left\|\nabla \xi_{k}\right\|_{B, 0, p} \leq\left\|F_{k}\right\|_{B, 0, p},
$$

letting $k \rightarrow+\infty$ yields (2.7).

Now we extend $\varphi$ to $\tilde{\varphi}$ on $B \backslash B_{\delta}\left(0<\delta<\frac{1}{2}\right)$ by

$$
\tilde{\varphi}(r, \theta):=\varphi(\theta), \quad \delta \leq r \leq 1, \theta \in \partial B .
$$

Choose a cut-off function $\eta$ such that $0 \leq \eta \leq 1$,

$$
\eta= \begin{cases}1 & r \geq \frac{3}{4} \\ 0 & r \leq \frac{1}{2}\end{cases}
$$

and $\left|\eta^{\prime}\right| \leq 2$, define

$$
\hat{\varphi}:=\eta \tilde{\varphi},
$$

then $\hat{\varphi} \in W^{1, p}\left(B, \Sigma^{n}\right)$ and $\psi-\hat{\varphi} \in W_{0}^{1, p}\left(B, \Sigma^{n}\right)$.

From (2.7), we have

$$
\begin{aligned}
\|\nabla(\psi-\hat{\varphi})\|_{B, 0, p} & \leq C\|\not(\psi-\hat{\varphi})\|_{B, 0, p} \\
& \leq C\left(\|f\|_{B, 0, p}+\|\not \hat{\varphi}\|_{B, 0, p}\right),
\end{aligned}
$$

which implies

$$
\|\nabla \psi\|_{B, 0, p} \leq C\left(\|f\|_{B, 0, p}+\|\not \hat{\varphi}\|_{B, 0, p}+\|\nabla \hat{\varphi}\|_{B, 0, p}\right) .
$$

Note that

$$
\begin{aligned}
\|\nabla \hat{\varphi}\|_{B, 0, p} & =\|\nabla(\eta \tilde{\varphi})\|_{B, 0, p} \\
& \leq C\left(\|\tilde{\varphi}\|_{B_{\frac{3}{4}} \backslash B_{\frac{1}{2}}, 0, p}+\|\nabla \tilde{\varphi}\|_{B \backslash B_{\frac{1}{2}}, 0, p}\right) \\
& \leq C\|\varphi\|_{\partial B, 0, p}+\left[\int_{B \backslash B_{\frac{1}{2}}}\left(\frac{1}{r}|\nabla \varphi|\right)^{p}\right]^{\frac{1}{p}} \\
& \leq C\left(\|\varphi\|_{\partial B, 0, p}+\|\nabla \varphi\|_{\partial B, 0, p}\right),
\end{aligned}
$$

namely,

$$
\|\nabla \hat{\varphi}\|_{B, 0, p} \leq C\|\varphi\|_{\partial B, 1, p} .
$$

Similarly,

$$
\|\not \hat{\varphi}\|_{B, 0, p} \leq C\|\varphi\|_{\partial B, 1, p} .
$$

Substituting (2.9) and (2.10) into (2.8) then yields:

$$
\|\nabla \psi\|_{B, 0, p} \leq C\left(\|f\|_{B, 0, p}+\|\varphi\|_{\partial B, 1, p}\right) .
$$

By the Poincaré inequality,

$$
\|\psi-\hat{\varphi}\|_{B, 0, p} \leq C\|\nabla(\psi-\hat{\varphi})\|_{B, 0, p},
$$


hence

$$
\|\psi\|_{B, 0, p} \leq C\|\nabla(\psi-\hat{\varphi})\|_{B, 0, p}+\|\hat{\varphi}\|_{B, 0, p},
$$

but

$$
\|\hat{\varphi}\|_{B, 0, p}=\|\eta \tilde{\varphi}\|_{B, 0, p} \leq C\|\varphi\|_{\partial B, 0, p},
$$

putting this and (2.9), (2.11) into (2.12), we finally obtain (2.4).

Q.E.D.

Now we can give the

Proof of Theorem 2.1 We first derive the following estimate:

$$
\|\psi\|_{B^{\prime}, 0, p} \leq C\|\psi\|_{B, 0,4},
$$

$\forall B^{\prime} \subset \subset B$, where $C=C\left(B^{\prime}, p\right)>0$ is a constant.

For this, we choose a cut-off function $\eta$ such that $0 \leq \eta \leq 1,\left.\eta\right|_{B^{\prime}} \equiv 1$, and supp $\eta \subset B$. Denote $\xi:=\eta \psi$, then

$$
\begin{aligned}
\not \partial \xi & =\not \partial(\eta \psi) \\
& =\eta \not \partial \psi+\nabla \eta \cdot \psi \\
& =\eta H_{j k l}\left\langle\psi^{j}, \psi^{k}\right\rangle \psi^{l}+\nabla \eta \cdot \psi .
\end{aligned}
$$

From Lemma 2.2, for any $1<q<2$,

$$
\begin{aligned}
\|\xi\|_{B, 1, q} & \leq C\left\|\eta H_{j k l}\left\langle\psi^{j}, \psi^{k}\right\rangle \psi^{l}+\nabla \eta \cdot \psi\right\|_{B, 0, q} \\
& \leq C\left(h_{0}\left\|\eta|\psi|^{3}\right\|_{B, 0, q}+\|\psi\|_{B, 0, q}\right) .
\end{aligned}
$$

Now observing that

$$
\begin{aligned}
\left\|\eta|\psi|^{3}\right\|_{B, 0, q} & =\left[\int_{B}\left(|\psi|^{2}|\eta \psi|\right)^{q}\right]^{\frac{1}{q}} \\
& =\left(\int_{B}\left(|\psi|^{2 q}|\xi|^{q}\right)\right)^{\frac{1}{q}} \\
& \leq\left(\int_{B}|\psi|^{4}\right)^{\frac{1}{2}}\left(\int_{B}|\xi|^{q^{*}}\right)^{\frac{1}{q^{*}}},
\end{aligned}
$$

where $q^{*}:=\frac{2 q}{2-q}$, putting (2.16) into (2.15), and using the Sobolev embedding, we have

$$
\|\xi\|_{B, 0, q^{*}} \leq C\left(h_{0}\|\psi\|_{B, 0,4}^{2}\|\xi\|_{B, 0, q^{*}}+\|\psi\|_{B, 0,4}\right) .
$$

Thus, if $\varepsilon>0$ is small enough such that $C h_{0} \sqrt{\varepsilon}<\frac{1}{2}$, then for $\psi$ with

$$
\int_{B}|\psi|^{4}<\varepsilon
$$

we have

$$
\|\xi\|_{B, 0, q^{*}} \leq C\|\psi\|_{B, 0,4} .
$$


Clearly, for any $p>1$, one can find some $q<2$ such that $p=q^{*}$. This establishes (2.13).

Next, since

$$
\begin{aligned}
\int_{B}|\nabla \xi|^{2} & =\int_{B}|\not \partial \xi|^{2} \\
& =\int_{B}|\eta \not \partial \psi+\nabla \eta \cdot \psi|^{2} \\
& \leq C\left(\int_{B}|\psi|^{6}+\int_{B}|\psi|^{2}\right),
\end{aligned}
$$

we have

$$
\begin{aligned}
\|\nabla \xi\|_{B, 0,2} & \leq C\left(\|\psi\|_{B, 0,6}^{3}+\|\psi\|_{B, 0,2}\right) \\
& \leq C\left(\|\psi\|_{B, 0,4}^{3}+\|\psi\|_{B, 0,4}\right) \\
& \leq C\|\psi\|_{B, 0,4}\left(1+\|\psi\|_{B, 0,4}^{2}\right) \\
& \leq C\|\psi\|_{B, 0,4},
\end{aligned}
$$

where in the second step we have used (2.13) and in the last step we have used (2.1). We then have,

$$
\|\nabla \psi\|_{B^{\prime}, 0,2} \leq C\|\psi\|_{B, 0,4},
$$

where $C>0$ is constant depending only on $h_{0}$ and $B^{\prime}$. Using the Weitzenböck formula and noting that the scalar curvature vanishes in this case, we have

$$
\begin{aligned}
\nabla_{\alpha} \nabla_{\alpha} \psi= & -\not{ }^{2} \psi \\
= & -\not \partial\left(H_{j k l}\left\langle\psi^{j}, \psi^{k}\right\rangle \psi^{l}\right) \\
= & -\left\langle\psi^{j}, \psi^{k}\right\rangle\left(\nabla H_{j k l} \cdot \psi^{l}\right)-H_{j k l}\left(\left\langle\nabla_{e_{\alpha}} \psi^{j}, \psi^{k}\right\rangle+\left\langle\psi^{j}, \nabla_{e_{\alpha}} \psi^{k}\right\rangle\right)\left(e_{\alpha} \cdot \psi^{l}\right) \\
& -H_{j k l} H_{p q r}^{l}\left\langle\psi^{j}, \psi^{k}\right\rangle\left\langle\psi^{p}, \psi^{q}\right\rangle \psi^{r} .
\end{aligned}
$$

Therefore, for any $\eta \in C^{\infty}(B)$,

$$
|\Delta(\eta \psi)| \leq C\left(|\psi|+|\nabla \psi|+|\nabla \psi||\psi|^{2}+|\psi|^{3}+|\psi|^{5}\right),
$$

where $C>0$ is a constant depending only on $\eta, h_{0}$ and $h_{1}$, from which we have

$$
\|\eta \psi\|_{2, p} \leq C\left(\|\psi\|_{0, p}+\|\nabla \psi\|_{0, p}+\left\|\left|\nabla \psi\left\|\left.\psi\right|^{2}\right\|_{0, p}+\left\||\psi|^{3}\right\|_{0, p}+\left\||\psi|^{5}\right\|_{0, p}\right) .\right.\right.
$$

Using the above estimates (2.13) and (2.17), we have

$$
\begin{aligned}
\left\|\left|\nabla \psi\left\|\left.\psi\right|^{2}\right\|_{B^{\prime}, 0, \frac{4}{3}}\right.\right. & \leq\|\nabla \psi\|_{B^{\prime}, 0,2}\|\psi\|_{B^{\prime}, 0,8}^{2} \\
& \leq C\|\psi\|_{B, 0,4}, \\
\left\||\psi|^{3}\right\|_{B, 0, p} & \leq C\|\psi\|_{B, 0,4} \\
\left\||\psi|^{5}\right\|_{B, 0, p} & \leq C\|\psi\|_{B, 0,4} .
\end{aligned}
$$

Substituting these into (2.18) on $B^{\prime}$ with $p=\frac{4}{3}$ and using (2.13), (2.17) again, we conclude that

$$
\|\eta \psi\|_{B^{\prime}, 2, \frac{4}{3}} \leq C\|\psi\|_{B, 0,4}
$$


This implies that

$$
\|\psi\|_{B^{\prime \prime}, 2, \frac{4}{3}} \leq C\|\psi\|_{B, 0,4}
$$

for any $B^{\prime \prime} \subset \subset B^{\prime}$. By Sobolev,

$$
\|\psi\|_{B^{\prime \prime}, 1,4} \leq C\|\psi\|_{B, 0,4},
$$

and consequently, $\|\psi\|_{L^{\infty}\left(B^{\prime \prime}\right)} \leq C\|\psi\|_{B, 0,4}$.

Choose $p=2$ in (2.18) and use the above estimates, we have

$$
\|\psi\|_{B^{\prime}, 2,2} \leq C\|\psi\|_{B, 0,4} .
$$

This yields

$$
\|\psi\|_{B^{\prime}, 1, p} \leq C\|\psi\|_{B, 0,4} .
$$

We can then obtain all the desired estimates by the standard bootstrap method, for example, using $\|\psi\|_{B^{\prime}, 1, p} \leq C\|\psi\|_{B, 0,4}$ in (2.18) we have $\|\psi\|_{B^{\prime \prime}, 2, p} \leq C\|\psi\|_{B, 0,4}$ for any $B^{\prime \prime} \subset \subset B^{\prime}$ and $p>1$.

Q.E.D.

From the above estimates, we have the following (see e.g. [12]):

Corollary 2.3 Let $M$ be a compact Riemannian surface without boundary, with a fixed spin structure. If $\psi \in W^{1, p}$ is a solution of

$$
\not \partial=F
$$

with $F \in L^{p}$, then

$$
\|\psi\|_{M, 1, p} \leq C\left(\|F\|_{M, 0, p}+\|\psi\|_{M, 0, p}\right),
$$

where $C=C(M, p)>0$ is a constant.

Proof Assume that $\left\{U_{\alpha}, \varphi_{\alpha}\right\}$ is a finite covering of $M$ by charts. Let $\left\{g_{\alpha}\right\}$ be a partition of unit subordinate to this covering. Denote $\psi_{\alpha}:=g_{\alpha} \psi$, and $F_{\alpha}:=\not \partial \psi_{\alpha}=g_{\alpha} F+\nabla g_{\alpha} \cdot \psi$, then in each chart $\left\{U_{\alpha}, \varphi_{\alpha}\right\}$, using (2.7) we have

$$
\left\|\psi_{\alpha}\right\|_{U_{\alpha}, 1, p} \leq C_{\alpha}\left(\left\|F_{\alpha}\right\|_{M, 0, p}+\left\|\psi_{\alpha}\right\|_{M, 0, p}\right),
$$

noting that $\psi=\sum_{\alpha} \psi_{\alpha},\left\|\nabla g_{\alpha}\right\| \leq C$ and $g_{\alpha}<1$, we obtain (2.20).

Q.E.D.

\section{Removable singularity theorem}

For a given smooth solution $\psi$ of (1.2) on the sphere $\mathbb{S}^{2}$, one can create a solution $\widetilde{\psi}$ on the Euclidean plane $\mathbb{R}^{2}$ through the stereographic projection from the north pole $N$, by virtue of the conformal invariance of the equation. Conversely, given a solution $\psi$ on $\mathbb{R}^{2}$, through the stereographic projection, we only have a solution $\psi$ on $\mathbb{S}^{2} \backslash\{N\}$, which then leads to the question of removable singularities. In this section, we will prove the following

Theorem 3.1 (Removable singularity theorem) Let $\psi$ be a solution of (1.2) which is smooth on $B \backslash\{0\}$. If

$$
\int_{B}|\psi|^{4}<\infty,
$$

then $\psi$ extends to a smooth solution of (1.2) on the whole B. 
Proof Since (1.2) is conformally invariant, by a rescaling transformation, we may assume that

$$
\int_{B}|\psi|^{4}<\varepsilon,
$$

where $\varepsilon>0$ is a small constant whose appropriate value will be determined later. For any given small $\delta>0$, we choose a cut-off function $\eta_{\delta} \in C_{0}^{\infty}\left(B_{2 \delta}\right)$ such that $0 \leq \eta_{\delta} \leq 1$,

$$
\eta_{\delta}=\left\{\begin{array}{lll}
1 & \text { in } & B_{\delta} \\
0 & \text { in } & B \backslash B_{2 \delta},
\end{array}\right.
$$

and $\left|\nabla \eta_{\delta}\right| \leq C / \delta$. Then

$$
\begin{aligned}
\not\left[\left[\left(1-\eta_{\delta}\right) \psi\right]\right. & =\left(1-\eta_{\delta}\right) \not \partial-\nabla \eta_{\delta} \cdot \psi \\
& =\left(1-\eta_{\delta}\right) H_{j k l}\left\langle\psi^{j}, \psi^{k}\right\rangle \psi^{l}-\nabla \eta_{\delta} \cdot \psi .
\end{aligned}
$$

By Lemma 2.2, we have

$$
\begin{aligned}
\left\|\left(1-\eta_{\delta}\right) \psi\right\|_{B, 1, \frac{4}{3} \leq} & C\left\|\left(1-\eta_{\delta}\right) H_{j k l}\left\langle\psi^{j}, \psi^{k}\right\rangle \psi^{l}-\nabla \eta_{\delta} \cdot \psi\right\|_{B, 0, \frac{4}{3}} \\
& +C\|\psi\|_{\partial B, 1, \frac{4}{3}} \\
\leq & C\left(h_{0}\|\psi\|_{B, 0,4}^{3}+\left\|\nabla \eta_{\delta} \cdot \psi\right\|_{B, 0, \frac{4}{3}}+\|\psi\|_{\partial B, 1, \frac{4}{3}}\right) .
\end{aligned}
$$

By the Sobolev embedding theorem, we have

$$
\left\|\left(1-\eta_{\delta}\right) \psi\right\|_{B, 0,4} \leq C\left(h_{0}\|\psi\|_{B, 0,4}^{3}+\left\|\nabla \eta_{\delta} \cdot \psi\right\|_{B, 0, \frac{4}{3}}+\|\psi\|_{\partial B, 1, \frac{4}{3}}\right) .
$$

We note that as $\delta \rightarrow 0$,

$$
\begin{aligned}
\left\|\nabla \eta_{\delta} \cdot \psi\right\|_{B, 0, \frac{4}{3}} & =\left(\int_{B_{2 \delta} \backslash B_{\delta}}\left|\nabla \eta_{\delta}\right|^{\frac{4}{3}}|\psi|^{\frac{4}{3}}\right)^{\frac{3}{4}} \\
& \leq \frac{C}{\delta}\left(\int_{B_{2 \delta}}|\psi|^{\frac{4}{3}}\right)^{\frac{3}{4}} \\
& \leq C\left(\int_{B_{2 \delta}}|\psi|^{4}\right)^{\frac{1}{4}} \rightarrow 0
\end{aligned}
$$

therefore, letting $\delta \rightarrow 0$ in (3.3) we obtain

$$
\|\psi\|_{B, 0,4} \leq C h_{0}\|\psi\|_{B, 0,4}^{2}\|\psi\|_{B, 0,4}+C\|\psi\|_{\partial B, 1, \frac{4}{3}} .
$$

We choose $\varepsilon>0$ so small that $C h_{0} \sqrt{\varepsilon}<1 / 2$, then

$$
\begin{aligned}
\|\psi\|_{B, 0,4} & \leq C\|\psi\|_{\partial B, 1, \frac{4}{3}} \\
& \leq C\left(\int_{\partial B}|\nabla \psi|^{\frac{4}{3}}\right)^{\frac{3}{4}}+C\left(\int_{\partial B}|\psi|^{4}\right)^{\frac{1}{4}} .
\end{aligned}
$$


By a rescaling argument, we have for any $r \in(0,1]$,

$$
\begin{aligned}
\left(\int_{B_{r}}|\psi|^{4}\right)^{\frac{1}{4}} & \leq C\left(r \int_{\partial B_{r}}|\nabla \psi|^{\frac{4}{3}}\right)^{\frac{3}{4}}+C\left(r \int_{\partial B_{r}}|\psi|^{4}\right)^{\frac{1}{4}} \\
\leq C & {\left[\left(r \int_{\partial B_{r}}|\nabla \psi|^{\frac{4}{3}}\right)^{\frac{1}{4}}+\left(r \int_{\partial B_{r}}|\psi|^{4}\right)^{\frac{1}{4}}\right], }
\end{aligned}
$$

that is,

$$
\int_{B_{r}}|\psi|^{4} \leq C r \int_{\partial B_{r}}|\nabla \psi|^{\frac{4}{3}}+C r \int_{\partial B_{r}}|\psi|^{4} .
$$

Denote

$$
\bar{\psi}:=\frac{1}{2 \pi} \int_{\partial B} \psi
$$

then on $B \backslash\{0\}$ :

$$
\not \partial(\psi-\bar{\psi})=H_{j k l}\left\langle\psi^{j}, \psi^{k}\right\rangle \psi^{l}=H_{j k l}\left\langle\psi^{j}, \psi^{k}\right\rangle\left(\psi^{l}-\bar{\psi}^{l}\right)+H_{j k l}\left\langle\psi^{j}, \psi^{k}\right\rangle \bar{\psi}^{l} .
$$

From Lemma 2.2, we have

$$
\begin{aligned}
\|\psi-\bar{\psi}\|_{B, 1, \frac{4}{3}} \leq & C\left(\left\|H_{j k l}\left\langle\psi^{j}, \psi^{k}\right\rangle\left(\psi^{l}-\bar{\psi}^{l}\right)\right\|_{B, 0, \frac{4}{3}}+\left\|H_{j k l}\left\langle\psi^{j}, \psi^{k}\right\rangle \bar{\psi}^{l}\right\|_{B, 0, \frac{4}{3}}\right. \\
& \left.+\|\psi-\bar{\psi}\|_{\partial B, 1, \frac{4}{3}}\right)
\end{aligned}
$$

using the Poincare's inequality, we obtain

$$
\begin{aligned}
\|\psi-\bar{\psi}\|_{B, 1, \frac{4}{3} \leq} & C\left(\left\|H_{j k l}\left\langle\psi^{j}, \psi^{k}\right\rangle\left(\psi^{l}-\bar{\psi}^{l}\right)\right\|_{B, 0, \frac{4}{3}}+\left\|H_{j k l}\left\langle\psi^{j}, \psi^{k}\right\rangle \bar{\psi}^{l}\right\|_{B, 0, \frac{4}{3}}\right. \\
& \left.+\|\nabla(\psi-\bar{\psi})\|_{\partial B, 0, \frac{4}{3}}\right) \\
\leq & C h_{0}\|\psi\|_{B, 0,4}^{2}\|\psi-\bar{\psi}\|_{B, 0,4}+C h_{0}\|\psi\|_{B, 0,4}^{2}\|\bar{\psi}\|_{B, 0,4} \\
& +C\|\nabla \psi\|_{\partial B, 0, \frac{4}{3}} \\
\leq & C h_{0}\|\psi\|_{B, 0,4}^{2}\|\psi-\bar{\psi}\|_{B, 1, \frac{4}{3}}+C h_{0}\|\psi\|_{B, 0,4}^{2}\|\psi\|_{\partial B, 0,1} \\
& +C\|\nabla \psi\|_{\partial B, 0, \frac{4}{3}},
\end{aligned}
$$

using the smallness of $\|\psi\|_{B, 0,4}$ again, we have

$$
\|\psi-\bar{\psi}\|_{B, 1, \frac{4}{3}} \leq C h_{0}\|\psi\|_{B, 0,4}^{2}\|\psi\|_{\partial B, 0,4}+C\|\nabla \psi\|_{\partial B, 0, \frac{4}{3}},
$$

therefore,

$$
\|\nabla \psi\|_{B, 0, \frac{4}{3}} \leq C h_{0}\|\psi\|_{B, 0,4}^{2}\|\psi\|_{\partial B, 0,4}+C\|\nabla \psi\|_{\partial B, 0, \frac{4}{3}},
$$

that is,

$$
\left(\int_{B}|\nabla \psi|^{\frac{4}{3}}\right)^{\frac{3}{4}} \leq C h_{0}\left(\int_{B}|\psi|^{4}\right)^{\frac{1}{2}}\left(\int_{\partial B}|\psi|^{4}\right)^{\frac{1}{4}}+C\left(\int_{\partial B}|\nabla \psi|^{\frac{4}{3}}\right)^{\frac{3}{4}} .
$$


It is then easy to see that

$$
\begin{aligned}
\int_{B}|\nabla \psi|^{\frac{4}{3}} & \leq C h_{0}^{\frac{4}{3}}\left(\int_{B}|\psi|^{4}\right)^{\frac{2}{3}}\left(\int_{\partial B}|\psi|^{4}\right)^{\frac{1}{3}}+C \int_{\partial B}|\nabla \psi|^{\frac{4}{3}} \\
& \leq \sigma \int_{B}|\psi|^{4}+\frac{C}{\sigma} \int_{\partial B}|\psi|^{4}+C \int_{\partial B}|\nabla \psi|^{\frac{4}{3}}
\end{aligned}
$$

where $\sigma>0$ is small constant, and $C>0$ is constant depending only on $h_{0}$. By rescaling again, we have

$$
\int_{B_{r}}|\nabla \psi|^{\frac{4}{3}} \leq \sigma \int_{B_{r}}|\psi|^{4}+\frac{C}{\sigma} r \int_{\partial B_{r}}|\psi|^{4}+C r \int_{\partial B_{r}}|\nabla \psi|^{\frac{4}{3}}
$$

Combining (3.4) and (3.5), we have

$$
\int_{B_{r}}|\psi|^{4}+\int_{B_{r}}|\nabla \psi|^{\frac{4}{3}} \leq C r\left(\int_{\partial B_{r}}|\psi|^{4}+\int_{\partial B_{r}}|\nabla \psi|^{\frac{4}{3}}\right) .
$$

Denote $F(r):=\int_{B_{r}}|\psi|^{4}+|\nabla \psi|^{\frac{4}{3}}$, then

$$
F(r) \leq \operatorname{CrF}^{\prime}(r)
$$

which implies that

$$
F(r) \leq F(1) r^{\frac{1}{C}}
$$

From this, it follows that $\psi \in W^{1, p}$ for some $p>4 / 3$, and then, by the standard bootstrap method, one can conclude the smoothness of $\psi$.

Q.E.D.

Remark When $\psi$ is the spinor representing a surface $M$ in $\mathbb{R}^{3}$ with mean curvature $H$, and $z=x+\mathrm{i} y$ is the parameterization of $M$, then the metric of $M$ is

$$
\mathrm{d} s^{2}=|\psi|^{4} \mathrm{~d} z \mathrm{~d} \bar{z}
$$

and the condition (3.1) means that $\left(M, \mathrm{~d} s^{2}\right)$ has finite area.

\section{Energy identity}

Let $M$ be a compact Riemann surface with a fixed spin structure. Given a sequence $\left\{\psi_{m}\right\}$ of solutions of (1.2) on $M$, if we assume it is uniformly bounded in $L^{p}(p>4)$, then the standard bootstrap method implies that $\left\{\psi_{m}\right\}$ is uniformly bounded in $C^{r}\left(r \in \mathbb{Z}_{+}\right)$. However, in the case of the $L^{4}$-norm, examples show that this compactness is no longer true. If $\left\{\psi_{m}\right\}$ converges to $\psi$ weakly in $L^{4}$, then in the limit we may encounter bubbling phenomenon; namely, by a rescaling argument and the previous removable singularity theorem, we may get some solutions on $\mathbb{S}^{2}$, and this causes an energy loss. Comparing to the well-known case of harmonic maps, one naturally asks whether the blow up set is finite and the energy identity holds. In view of the Weierstrass representation, this corresponds to the question of the convergence of surfaces with a uniform area bound.

We will need the following lower bound for the energy of the bubbles: 
Lemma 4.1 There exists a constant $A>0$ such that for any nontrivial solution $\psi$ of (1.2) on $\mathbb{S}^{2}$, we have

$$
\int_{\mathbb{S}^{2}}|\psi|^{4} \geq A
$$

Proof First, for any solution $\psi$ on $\mathbb{S}^{2}$,

$$
\|\psi\|_{\frac{4}{3}} \leq C\|\not \partial \psi\|_{\frac{4}{3}} .
$$

Otherwise, for any $k \in \mathbb{Z}_{+}$, there is a $\psi_{k}$ which solves (1.2), but

$$
\left\|\psi_{k}\right\|_{\frac{4}{3}}>k\left\|\not \partial \psi_{k}\right\|_{\frac{4}{3}} \text {. }
$$

Denote $\eta_{k}:=\psi_{k} /\left\|\psi_{k}\right\|_{\frac{4}{3}}$, then

$$
\left\|\not \partial \eta_{k}\right\|_{\frac{4}{3}}<1 / k, \quad\left\|\eta_{k}\right\|_{\frac{4}{3}}=1 .
$$

Using Corollary 2.3, we have

$$
\left\|\eta_{k}\right\|_{1, \frac{4}{3}} \leq C\left(\left\|\not \partial \eta_{k}\right\|_{\frac{4}{3}}+\left\|\eta_{k}\right\|_{\frac{4}{3}}\right) \leq C
$$

which implies that there exists some $\eta_{0}$ such that $\eta_{k}$ converges to $\eta_{0}$ weakly in $W^{1, \frac{4}{3}}$. By Sobolev, $\eta_{k} \rightarrow \eta_{0}$ in $L^{\frac{4}{3}}$, so $\left\|\eta_{0}\right\|_{\frac{4}{3}}=\lim _{k \rightarrow \infty}\left\|\eta_{k}\right\|_{\frac{4}{3}}=1$. But $\not \partial \eta_{k}$ converges to $\not \partial \eta_{0}$ weakly in $L^{\frac{4}{3}}$, and from (4.3), it is easy to see that $\not \partial \eta_{0}=0$, hence $\eta_{0} \equiv 0$ since there is no nontrivial harmonic spinor on $\mathbb{S}^{2}$. This contradicts $\left\|\eta_{0}\right\|_{\frac{4}{3}}=1$.

Now from (4.2) and (2.20), we have

$$
\begin{aligned}
\|\psi\|_{1, \frac{4}{3}} & \leq C\left(\|\not \psi\|_{\frac{4}{3}}+\|\psi\|_{\frac{4}{3}}\right) \\
& \leq C\|\not \partial\|_{\frac{4}{3}} \\
& \leq C h_{0}\|\psi\|_{4}^{3},
\end{aligned}
$$

therefore,

$$
\|\psi\|_{4} \leq C h_{0}\|\psi\|_{4}^{3},
$$

and if $\|\psi\|_{4}$ is so small that $C h_{0}\|\psi\|_{4}^{2}<1$, then we have $\psi \equiv 0$. Equivalently, we can find a constant $A>0$ such that for any nontrivial solution $\psi$ of (1.2) on $\mathbb{S}^{2}$, the energy $\int_{M}|\psi|^{4}$ is bounded below by $A$.

Q.E.D.

Let

$$
S:=\cap_{r>0}\left\{\left.x \in M\left|\liminf _{m \rightarrow+\infty} \int_{B(x, r)}\right| \psi_{m}\right|^{4} \geq \varepsilon\right\}
$$

be the blow up set of $\left\{\psi_{m}\right\}$, where $\varepsilon$ is as in Theorem 2.1.

Theorem 4.2 (Energy identity) Let $M$ be a compact Riemann surface with fixed spin structure, and suppose that $\left\{\psi_{m}\right\}$ is a sequence of smooth solutions of (1.2) on M satisfying

$$
E\left(\psi_{m}\right):=\int_{M}\left|\psi_{m}\right|^{4} \leq \Lambda<+\infty .
$$


If $\left\{\psi_{m}\right\}$ converges to $\psi$ weakly in $L^{4}(M)$ (but not strongly,) then the (non-empty) blow up set $S$ must be finite:

$$
S=\left\{p_{1}, p_{2}, \ldots, p_{K}\right\} .
$$

Furthermore, there exists a constant $c_{0}>0$ depending only on $M$ such that if

$$
\sup _{M, i, j, k, l}\left|H_{j k l}^{i}\right| \sqrt{\Lambda}<c_{0},
$$

then the energy identity for $\left\{\psi_{m}\right\}$ holds, namely, for each blow up point $p_{k}(k=1,2, \ldots, K)$, there exist a finite number of solutions $\left\{\xi_{k}^{a}\right\}_{a=1,2, \cdots, A_{k}}$ of (1.2) on $\mathbb{S}^{2}$ such that

$$
\lim _{n \rightarrow+\infty} E\left(\psi_{m}\right)=E(\psi)+\sum_{k=1}^{K} \sum_{a=1}^{A_{k}} E\left(\xi_{k}^{a}\right) .
$$

Proof Since the removable singularity theorem, the small energy regularity theorem, and Lemma 4.1 provide key ingredients for establishing the energy identity, the theorem can then be proved by an argument as the proof of Theorem 3.6 in [5], see also [7]. Here we only give a sketch of proof.

First, the condition $E\left(\psi_{m}\right) \leq \Lambda<+\infty$ and Theorem 2.1 imply that the blow up set $S$ must be finite. We choose small disks $B_{\delta_{k}}$ for each $p_{k}$ such that $B_{\delta_{k}} \cap B_{\delta_{j}}=\phi$ for $k \neq j, k, j=1,2, \ldots, K$. Furthermore, by Theorem $2.1,\left\{\psi_{m}\right\}$ strongly converges to $\psi$ in $L^{4}$ on $M \backslash \cup_{k=1}^{K} B_{\delta_{k}}$, (4.6) is then equivalent to

$$
\sum_{k=1}^{K} \lim _{\delta_{k} \rightarrow 0} \lim _{n \rightarrow \infty} E\left(\psi_{m} ; B_{\delta_{k}}\right)=\sum_{k=1}^{K} \sum_{a=1}^{A_{k}} E\left(\xi_{k}^{a}\right) .
$$

It suffices to prove that for each blow-up point $p$, we have

$$
\lim _{\delta \rightarrow 0} \lim _{m \rightarrow \infty} E\left(\psi_{m} ; B_{\delta}\right)=\sum_{a=1}^{A} E\left(\xi^{l}\right) .
$$

By virtue of the conformal invariance of the equation (1.2), and the locality of the problem, we may assume that each disk $B_{\delta}$ is equipped with the Euclidean metric. For each $\psi_{m}$, we choose $\lambda_{m}$ and $x_{m} \in B_{\delta}$ such that $\lambda_{m} \rightarrow 0, x_{m} \rightarrow p$ and

$$
E\left(\psi_{m} ; B_{\lambda_{m}}\left(x_{m}\right)\right)=\max _{x \in B_{\delta}(p)} E\left(\psi_{m} ; B_{\lambda_{m}}(x)\right)=\frac{\varepsilon}{2} .
$$

Rescaling by

$$
\widetilde{\psi}_{m}(x):=\lambda_{m}^{-\frac{1}{2}} \psi_{m}\left(x_{m}+\lambda_{m} x\right),
$$

then

$$
\begin{gathered}
E\left(\widetilde{\psi}_{m} ; B\right)=E\left(\psi_{m} ; B_{\lambda_{m}}\left(x_{m}\right)\right)=\frac{\varepsilon}{2}<\varepsilon, \\
E\left(\widetilde{\psi}_{m} ; B_{R}\right)=E\left(\psi_{m} ; B_{\lambda_{m} R}\left(x_{m}\right)\right) \leq \Lambda .
\end{gathered}
$$

By Theorem 2.1, we have a subsequence of $\left\{\psi_{m}\right\}$ which strongly converges to some $\widetilde{\psi}$ in $L^{4}\left(B_{R}\right)$ for any $R \geq 1$. We thus obtain a nonconstant solution $\widetilde{\psi}$ of (1.2) on $\mathbb{R}^{2}$, and hence a nonconstant solution $\xi^{1}$ of (1.2) on $\mathbb{S}^{2} \backslash\{N\}$ with bounded energy. Theorem 3.1 then gives 
us a nonconstant solution of (1.2) on the whole $\mathbb{S}^{2}$, and we obtain the first bubble $\xi^{1}$ at the blow up point $p$.

Next, denote

$$
A(\delta, R, m):=\left\{x \in \mathbb{R}^{2}\left|\lambda_{m} R \leq\right| x-x_{m} \mid \leq \delta\right\},
$$

then (4.8) is equivalent to

$$
\lim _{R \rightarrow \infty} \lim _{\delta \rightarrow 0} \lim _{m \rightarrow \infty} E\left(\psi_{m} ; A(\delta, R, m)\right)=\sum_{a=2}^{A} E\left(\xi^{a}\right) .
$$

For a fixed blow-up point $p$, the number of bubbles $\xi$ must be finite; this follows easily from Lemma 4.1. We only consider (4.9) in the case of exactly one bubble at the blow up point $p$, because the case of at least two bubbles can be reduced to this case. Then, (4.9) is just

$$
\lim _{R \rightarrow \infty} \lim _{\delta \rightarrow 0} \lim _{m \rightarrow \infty} E\left(\psi_{m} ; A(\delta, R, m)\right)=0 .
$$

In order to prove this, we consider a conformal transformation $f: \mathbb{R} \times \mathbb{S}^{1} \rightarrow \mathbb{R}^{2}, f(t, \theta)=$ $\left(e^{-t}, \theta\right)$, where $\mathbb{R} \times \mathbb{S}^{1}$ is given the metric $g=\mathrm{d} t^{2}+\mathrm{d} \theta^{2}$. for the pull-back $\Psi_{m}:=f^{*} \psi_{m}$, then $E\left(\Psi_{m}\right) \leq \Lambda$. Set $T_{0}:=|\log \delta|, T_{m}:=\left|\log \lambda_{m} R\right|$.

Using Theorem 3.1, through an argument by contradiction (c.f. p. 82 in [5] for more details), one can prove that there is a $K>0$ such that if $m \geq K$, then

$$
\int_{[t, t+1] \times \mathbb{S}^{1}}\left|\Psi_{m}\right|^{4}<\varepsilon, \quad \forall t \in\left[T_{0}, T_{m}-1\right] .
$$

Choose a cut-off function $\eta$ on $B\left(x_{m}, 2 \delta\right)$ as follows:

$$
\begin{gathered}
\eta \in C_{0}^{\infty}\left(B_{2 \delta} \backslash B_{\lambda_{m} R / 2}\right) ; \quad \eta \equiv 1 \quad \text { in } \quad B_{\delta} \backslash B_{\lambda_{m} R} \\
|\nabla \eta| \leq C / \delta \text { in } B_{2 \delta} \backslash B_{\delta} ; \quad|\nabla \eta| \leq C / \lambda_{m} R \quad \text { in } \quad B_{\lambda_{m} R} \backslash B_{\lambda_{m} R / 2},
\end{gathered}
$$

where we denote $B_{\delta}:=B\left(x_{m}, \delta\right)$ etc. for simplicity. Then from Lemma 2.2 we have

$$
\begin{aligned}
\left\|\eta \psi_{m}\right\|_{L^{4}} & \leq C\left\|\eta \not \partial \psi_{m}+\nabla \eta \cdot \psi_{m}\right\|_{L^{\frac{4}{3}}} \\
& \leq C\left\|h _ { 0 } \left|\eta \left\|\left.\psi_{m}\right|^{3}+\left|\nabla \eta\left\|\psi_{m} \mid\right\|_{L^{\frac{4}{3}}}\right.\right.\right.\right. \\
& \leq C h_{0}\left\|\psi_{m}\right\|_{L^{4}}^{2}\left\|\eta \psi_{m}\right\|_{L^{4}}+C\left[\int_{A(2 \delta, R / 2, m)}\left(\left|\nabla \eta \| \psi_{m}\right|\right)^{\frac{4}{3}}\right]^{\frac{3}{4}} \\
& \leq C h_{0} \sqrt{\Lambda}\left\|\eta \psi_{m}\right\|_{L^{4}}+C\left[\int_{A(2 \delta, R / 2, m)}\left(\left|\nabla \eta \| \psi_{m}\right|\right)^{\frac{4}{3}}\right]^{\frac{3}{4}} .
\end{aligned}
$$

Clearly, there exists a constant $c_{0}>0$ such that when (4.5) is satisfied, we have $C h_{0} \sqrt{\Lambda}<1$, from the above estimate, we then have

$$
\left\|\eta \psi_{m}\right\|_{L^{4}} \leq C\left[\int_{B_{2 \delta} \backslash B_{\delta}}\left(|\nabla \eta|\left|\psi_{m}\right|\right)^{\frac{4}{3}}\right]^{\frac{3}{4}}+C\left[\int_{B_{\lambda_{m} R} \backslash B_{\lambda_{m} R / 2}}\left(\left|\nabla \eta \| \psi_{m}\right|\right)^{\frac{4}{3}}\right]^{\frac{3}{4}} .
$$


Therefore,

$$
\begin{aligned}
\left\|\psi_{m}\right\|_{L^{4}(A(\delta, R, m))} & \leq C\left[\int_{B_{2 \delta} \backslash B_{\delta}}\left|\psi_{m}\right|^{4}\right]^{\frac{1}{4}}+C\left[\int_{B_{\lambda_{m} R} \backslash B_{\lambda_{m} R / 2}}\left|\psi_{m}\right|^{4}\right]^{\frac{1}{4}} \\
& \leq C \varepsilon^{\frac{1}{4}}+C \varepsilon^{\frac{1}{4}},
\end{aligned}
$$

where in the last step, we used (4.11). This proves (4.10).

Q.E.D.

\section{Related types of Dirac equations}

Let $M$ be a compact Riemann surface with fixed spin structure. For any local orthonormal basis $\left\{e_{\alpha}\right\}_{\alpha=1,2}$, one can define the so-called chirality operator

$$
\Gamma:=i e_{1} \cdot e_{2} \cdot
$$

This definition is independent of the choice of $\left\{e_{\alpha}\right\}_{\alpha=1,2}$, therefore, $\Gamma$ is globally defined on $M$. Define

$$
\Gamma_{+}:=\frac{1}{2}(I d+\Gamma), \quad \Gamma_{-}:=\frac{1}{2}(I d-\Gamma) .
$$

Let $U=U(\psi), V=V(\psi)$ be complex functions. We consider the following Dirac equation:

$$
\not \partial \psi=\left[U(\psi) \Gamma_{+}+V(\psi) \Gamma_{-}\right] \psi .
$$

Clearly, (1.2) corresponds to the case $U=V=-H|\psi|^{2}$. Comparing to (1.2), the Dirac equation for surfaces immersed into some three-dimensional Lie group $N$ takes a special form of (5.1). For example (c.f. Sect. 2.3 in [16])

$$
\begin{gathered}
N=S U(2): \quad U=\bar{V}=-(H-i)|\psi|^{2} \\
N=N i l: \quad U=V=-H|\psi|^{2}-\frac{i}{2}\left(\left|\psi_{1}\right|^{2}-\left|\psi_{2}\right|^{2}\right) ; \\
N=\widetilde{S L_{2}}: \quad U=-H|\psi|^{2}-i\left(\frac{3}{2}\left|\psi_{2}\right|^{2}-\left|\psi_{1}\right|^{2}\right), \\
V=-H|\psi|^{2}-i\left(\left|\psi_{2}\right|^{2}-\frac{3}{2}\left|\psi_{1}\right|^{2}\right) .
\end{gathered}
$$

Using the same methods as in the previous sections, one can conclude similar results for the above types of Dirac equations.

Corollary 5.1 There exists a small constant $\varepsilon>0$ such that for any smooth solution $\psi$ of (5.1), with $U, V$ satisfying one of (5.2), (5.3), (5.4), and

$$
E(\psi ; B):=\int_{B}|\psi|^{4}<\varepsilon,
$$

we have

$$
\|\psi\|_{B^{\prime}, k, p} \leq C\|\psi\|_{B, 0,4},
$$


$\forall B^{\prime} \subset \subset B, 1<p$ and $k \in \mathbb{Z}_{+}$, where $C=C\left(B^{\prime}, k, p\right)>0$ is constant, and $\|\cdot\|_{B, k, p}$ denotes the norm in $W^{k, p}\left(B, \Sigma^{n}\right)$.

Corollary 5.2 Let $\psi$ be a solution of (5.1), with $U, V$ satisfying one of (5.2), (5.3), (5.4), which is smooth on $B \backslash\{0\}$. If

$$
\int_{B}|\psi|^{4}<\infty
$$

then $\psi$ extends to a smooth solution on the whole $B$.

Corollary 5.3 Let $M$ be a compact Riemann surface with fixed spin structure, and suppose that $\left\{\psi_{m}\right\}$ is a sequence of smooth solutions of (5.1), with $U, V$ satisfying one of (5.2), (5.3), (5.4) respectively, on $M$ and

$$
E\left(\psi_{m}\right):=\int_{M}\left|\psi_{m}\right|^{4} \leq \Lambda<+\infty .
$$

If $\left\{\psi_{m}\right\}$ converges to $\psi$ weakly in $L^{4}(M)$ (but not strongly), then the (non-empty) blow up set $S$ must be finite:

$$
S=\left\{p_{1}, p_{2}, \ldots, p_{K}\right\} .
$$

Furthermore, there exists a constant $c_{0}>0$ depending only on $M$ such that if

$$
\left(\sup _{M}|H|+\alpha\right) \sqrt{\Lambda}<c_{0},
$$

with $\alpha=1, \frac{1}{2}, \frac{3}{2}$ respectively, then the energy identity for $\left\{\psi_{m}\right\}$ holds, namely, for each blow up point $p_{k}(k=1,2, \ldots, K)$, there exist a finite number of solutions $\left\{\xi_{k}^{a}\right\}_{a=1,2, \ldots, A_{k}}$ of $(5.1)$ on $\mathbb{S}^{2}$ such that

$$
\lim _{m \rightarrow+\infty} E\left(\psi_{m}\right)=E(\psi)+\sum_{k=1}^{K} \sum_{a=1}^{A_{k}} E\left(\xi_{k}^{a}\right) .
$$

Acknowledgements The research of QC is partially supported by NSFC (Grant No.10571068) and SRF for ROCS, SEM; he also thanks the Max Planck Institute for Mathematics in the Sciences for good working conditions during his visit.

\section{References}

1. Amman, B.: A variational problem in conformal spin geometry. Habilitationsschrift, Universität Hamburg, May 2003, http://www.berndammann.de/publications

2. Ammann, B., Humbert, E.: The first conformal Dirac eigenvalue on 2-dimensional tori. J. Geom. Phys. 56(4), 623-642 (2006).

3. Bartnik, R., Chruściel, P.T.: Boundary value problems for Dirac-type equations. J. Reine Angew. Math. 579, 13-73 (2005)

4. Chen, Q., Jost, J., Li, J.Y., Wang, G.F.: Dirac-harmonic maps. Math. Z. 254, 409-432 (2006)

5. Chen, Q., Jost, J., Li, J.Y., Wang, B.: Regularity theorem and energy identities for Dirac-harmonic maps. Math. Z. 251, 61-84 (2005)

6. Chen, Q., Jost, J., Wang, G.F.: Liouville theorems for Dirac-harmonic maps. Preprint 2006

7. Ding, W.Y., Tian, G.: Energy identity for a class of approximate harmonic maps from surfaces. Commun. Anal. Geom. 3, 543-554 (1996)

8. Friedrich, T.: On the spinor representation of surfaces in Euclidean 3-space. J. Geom. Phy. 28, 143-157 (1998) 
9. Gilberg, D., Trudinger, N.: Elliptic Partial Differential Equations of Second Order. Springer-Verlag (1998)

10. Jost, J.: Two-Dimensional Geometric Variational Problems. Wiley (1991)

11. Kenmotsu, K.: Weierstrass formula for surfaces of prescribed mean curvature. Math. Ann. 245, 89-99 (1979)

12. Mcduff, D., Salamon, D.: J-Holomorphic Curves and Quantum Cohomology. AMS Providence, Rhode Island (1994)

13. Parker, T.H.: Bubble tree convergence for harmonic maps. J. Diff. Geom. 44(3), 595-633 (1996)

14. Parker, T.H., Wolfson, J.G.: Pseudo-holomorphic maps and Bubble trees. J. Geom. Anal. 3, 63-98 (1993)

15. Sacks, J., Uhlenbeck, K.: The existence of minimal immersions of 2-spheres. Ann. Math. 113(1), $1-24(1981)$

16. Taimanov, I.S.: Two dimensional Dirac operator and surface theory. Russian Math. Surveys 61(1), 79-159 (2006) Math. Rev. MR2239773

17. Ye, R.: Gromov's compactness theorem for pseudo-holomorphic curves. Trans. Am. Math. Soc. 342, 671-694 (1994) 